\title{
Andrea Lindmayr-Brandl
}

\section{Das Alte und das Neue Agricolas »Fortuna desperata « im Interpretationsvergleich}

Als der Musiker Ulrich Brätel 1534 den Auftrag bekommt, für den wieder eingesetzten Herzog Ulrich von Württemberg ein Repertoire für dessen Hofkapelle zu sammeln, sucht er nicht nur nach Neuem. Er besinnt sich auch des >Guten Alten< und der damals schon >Alten Großen Meister<, wie er uns in dem von ihm vertonten Text wissen lässt: ${ }^{1}$

\author{
So ich betracht und acht der Alten G'sang, \\ mit Dank will ich ihr' Kunst hoch preisen: \\ Denn Ockeghem - fürnem - ist sehr kunstreich, \\ dergleichen thut Larue beweisen. \\ Sein' scharfen Sinn, Josquin acht ich subtil, \\ und will des Fincken Kunst auch rühmen, \\ [ge]braucht seltzam art, verkärth, auf frembd manier \\ wie schier thut Alexander führen.
}

Der »alte Gsang«, der hier geschätzt und geachtet wird, war damals schon rund fünfzig Jahre alt. Fünfhundert Jahre später befinden wir es in deutschen Landen erneut wert, uns mit dieser hoch gepriesenen »Kunst« eingehend zu beschäftigen. Von den fünf genannten Komponisten - Johannes Ockeghem, Pierre de La Rue, Josquin Desprez, Heinrich Finck und Alexander Agricola - interessiert uns insbesondere der Letztgenannte, seine »seltsame Art, das Verkehrte und die fremde Manier« in seiner Musik.

\section{Theorie: Zum Verhältnis des Vokalen zum Instrumentalen um 1500}

Das Aufkommen von Neuem ist immer mit Turbulenzen verbunden. Selten löst das Neue das Alte kurzerhand ab, in den meisten Fällen laufen beide

1 Das Lied ist überliefert in einem Druck von 1536 (Fünfvnd sechzig teütscher Lieder/ vormals $i \bar{m}$ truck nie $v \beta$ gangen, Straßburg: Schöffer \& Apiarius; RISM [1536 $]^{8}, \mathrm{Nr} .63$ ) und in einer Neuausgabe greifbar: 65 Deutsche Lieder firr vier-bis finfstimmigen gemischten Chor a cappella nach dem Liederbuch von Peter Schöffer und Mathias Apiarius (Biener) (Straßburg spätestens 1536). Erste Partiturausgabe, hrsg. von Hans Joachim Moser, Wiesbaden 1967, S. 207-211. Die sprachlich leicht modernisierte Textversion stammt von mir, der Originaltext kann bei Moser nachgelesen werden. 
Erscheinungen eine Zeitlang parallel nebeneinander her und beeinflussen sich gegenseitig. Dabei ist es in der Regel so, dass das Neue zunächst das Alte imitiert und möglichst auf dem bereits erreichten hohen Niveau anschließt und fortsetzt. Erst nach einer Weile gewinnt das Neue eigenständigen Charakter und wirkt nun umgekehrt auf das Alte zurück, das dadurch wiederum neue Impulse gewinnt.

Was ich hier über >das Alte und >das Neue $<$ gesagt habe, kann auf viele unterschiedliche Phänomene des kulturellen Wandels angewendet werden: etwa auf die Rolle von Gedächtnis und Schrift in der musikalischen Überlieferung, auf die Produktion von Musikhandschriften und den frühen Notendruck, aber auch auf den Instrumentenbau, wenn durch technische Neuerungen neue Instrumente aufkommen. In unserem Fall ist das Alte - grob gesprochen - die »a cappella«-Vokalmusik, das Neue die eigenständige Instrumentalmusik. Differenzierter betrachtet hat es natürlich immer schon Instrumentalmusik neben dem Vokalen gegeben. Allerdings war der Stellenwert dieser beiden Musizierweisen sehr unterschiedlich, und so geht es im Folgenden genauer gesagt um das Hervortreten von instrumentaler Musik gegenüber der bislang in der Kunstmusik so dominierenden Vokalmusik am Beginn des 16. Jahrhunderts.

In diesem Zeitraum gibt es in den überlieferten Dokumenten erstmals deutliche Hinweise dafür, dass das Musizieren mit Instrumenten eine gehobene Öffentlichkeit gefunden hat. Vorworte in gedruckten Sammlungen sprechen von variablen Besetzungsmöglichkeiten, die Benutzer solcher Sammlungen notieren gelegentlich die bevorzugte instrumentale Besetzung in den einzelnen Stimmbüchern, und von einigen wenigen Quellen wissen wir, dass sie schon im Hinblick auf ein bestimmtes Instrumentalensemble zusammengestellt wurden. Tabulaturschriften werden als Lern- und Spielhilfe entwickelt und lassen durch diese spezielle Aufzeichnungsweise erstmals eine eindeutige Trennung von vokaler und instrumentaler Musik zu. Dazu kommen zahlreiche Abbildungen aus der Zeit, die uns dieses Nebeneinander von Sängern, Lautenisten, Bläsern, Streichern, Harfenisten und Clavieristen anschaulich vor Augen führen. Schließlich haben sich auch vereinzelt Besetzungslisten von Hofkapellen erhalten, aus denen deutlich wird, dass Instrumentalisten sowohl solistisch als auch im Ensemble eine immer größere Rolle beigemessen wurde.

Mit dieser kurzen Rundumschau habe ich die soziale Situation in der Welt der Musik um die Jahrhundertwende angesprochen. Das ist aber nur eine Seite der Medaille. Die andere Seite ist die Frage, inwieweit die zeitgenössischen Komponisten auf diese Veränderungen musikalisch reagiert haben. Soziale Umbrüche durch den Notendruck, die neuen musikalischen Möglichkeiten, die die Instrumente boten und nun auch in den Denkhorizont der Schöpfer 
der artifiziellen Musik traten, haben die traditionellen Grenzen erweitert und nicht nur speziell für Instrumente gedachte Werke ans Licht gebracht. Sie haben auch umgekehrt auf die Vokalmusik zurückgewirkt und einen neuen »instrumentalen« Stil aufkommen lassen, der uns dazu verleitet, sie als Ganzes in den Bereich der Instrumentalkompositionen zu verweisen. Eine solche Annahme liegt besonders nahe, wenn die Worte in der überlieferten Quelle fehlen, denn - was sollte man da singen? Diese Diskussion ist in unserem Fach schon des Langen und des Breiten geführt worden und soll hier nicht wieder aufgegriffen werden. Ein grundlegendes Missverständnis dabei ist meiner Ansicht nach die zwanghafte Dichotomie zwischen Vokal- und Instrumentalmusik, die wir aus den nachfolgenden Jahrhunderten >geerbt $<$ haben. Meine These ist, dass es zu dieser Zeit ein interessantes Nebeneinander gibt, oder besser gesagt: ein Miteinander von beiden Musizierformen, die wir gar nicht dezidiert unterscheiden müssen. Und dieses $>$ Miteinander $<$ hat auch in der Kompositionstechnik seine Spuren hinterlassen.

Alexander Agricolas Werk ist dafür geradezu ein Paradebeispiel. Früher als andere Musiker seiner Generation nimmt er auf diese Entwicklung in seinen Kompositionen deutlichen Bezug. Seine Stücke wirken für uns »instrumental«, was so viel heißt wie: für das Singen nicht wirklich geeignet. Warum? Weil viele kleine Notenwerte die schlichte Linie verunklaren, rhythmische Finessen die Sänger fordern und die melodischen Linien nicht zielgerichtet, sondern satemlos< oder in langen Tonleitertiraden gestaltet sind. Dazu kommen große Intervallsprünge, ausnotierte Verzierungen und oftmals ein fehlender Text.

Gerade weil bei Agricola dieses instrumentale Komponieren so früh einsetzt - im Wesentlichen ist sein Schaffen noch vor der Jahrhundertwende entstanden -, und gerade weil dieses so stark ausgeprägt ist, scheint es bei ihm besonders interessant zu sein, Interpretationen in verschiedenen Besetzungen - rein vokal, gemischt vokal und instrumental, sowie rein instrumental - vergleichend zu hören. Ziel meines Beitrags ist es, die breiten und variablen klanglichen Erscheinungsformen einer Komposition von Agricola als Realität wahrzunehmen. Denn diese sind nicht zufällig oder aus Mangel an geeigneten Musikern entstanden, sondern wurden vom Komponisten mit einkalkuliert und waren somit bereits Teil des Konzepts.

\section{Praxis: Agricola und »Fortuna desperata«}

»Fortuna desperata « war ein überaus beliebtes italienisches Lied, das ein dreiviertel Jahrhundert lang immer wieder neu in Musik gesetzt wurde. 
Ausgehend von seinem Entstehungsort Florenz gelangte es früh in die musikalischen Zentren Neapel und Ferrara. Neben seiner Realisation als drei- bis fünfstimmiges weltliches Lied wanderte es sozusagen auch durch die Gattungen, indem es mehrmals als Grundlage von Messensätzen diente und für Tasteninstrumente bearbeitet wurde. Die $>$ Urgestalt $<-$ gemeint ist die früheste erhaltene mehrstimmige Vertonung - ist eine dreistimmige simple italienische Canzona in mehreren Strophen, von einem unbestimmten Komponisten. Lange war Antoine Busnoys als Autor im Verdacht, in jüngerer Zeit hat man mehrere Gegenargumente für eine Zuschreibung an ihn gefunden und lässt die Autorschaft lieber offen. ${ }^{2}$

Honey Meconi, die sich intensiv mit der Bearbeitung von beliebten Liedmelodien im 15. und 16. Jahrhundert beschäftigt hat, kann in einer von ihr herausgegebenen Notenedition 36 Liedvertonungen von »Fortuna desperata « präsentieren, die die verschiedensten Kompositionstechniken verwenden. ${ }^{3}$ Agricolas Beitrag dazu gehört zu den »si placet«-Kompositionen, wobei die künstlerische Leistung darin besteht, dem dreistimmigen >Ursatz< weitere Stimmen hinzuzufügen. Von den insgesamt fünf Nummern dieser Gruppe ergänzen vier anonym gebliebene Komponisten eine einzelne Stimme, indem sie zwischen Superius und Tenor einen Altus einschieben. Agricola hingegen erweitert den Stimmensatz gleich auf das Doppelte, fügt also zum bestehenden dreistimmigen Satz nicht weniger als drei weitere Stimmen hinzu: einen Contra-Altus zwischen Superius und Tenor, sowie einen Vagans und einen Bassus, im Ambitus beide unterhalb der ursprünglichen Bassstimme (nun »Baricanor « genannt) gelegen.

Die Funktion dieser >Ergänzungsstimmen lässt sich schon aus den ersten Takten ersehen (siehe Notenbeispiel 1). Der Contra-Altus ist typische Füllstimme, der klanglich zwischen Superius und Tenor vermittelt und Kadenzpassagen durch rhythmisch differenzierte Figuren überbrückt. Die beiden Unterstimmen haben zum einen eine erweiterte Bassfunktion, indem sie die Oktave unterhalb der ursprünglichen Bassus-Stimme aufbauen. Zum anderen sind sie auch freie Stimmen und nützen diese Funktion durch verspielte, oft verzahnte Einwürfe in kleinen, rhythmisch bewegten sinstrumentalen<

2 Martin Picker, »Henricus Isaac and Fortuna desperata «, in: Antoine Busnoys. Method, Meaning, and Context in Late Medieval Music, hrsg. von Paula Higgins, Oxford 1999, S. 431-445; Joshua Rifkin, »Busnoys and Italy: The Evidence of Two Songs«, in: ebda., S. 505-571.

3 Fortuna desperata. Thirty-Six Settings of an Italian Song, hrsg. von Honey Meconi, Madison 2001 (Recent Researches in the Music of the Middle Ages and Early Renaissance, 37). 

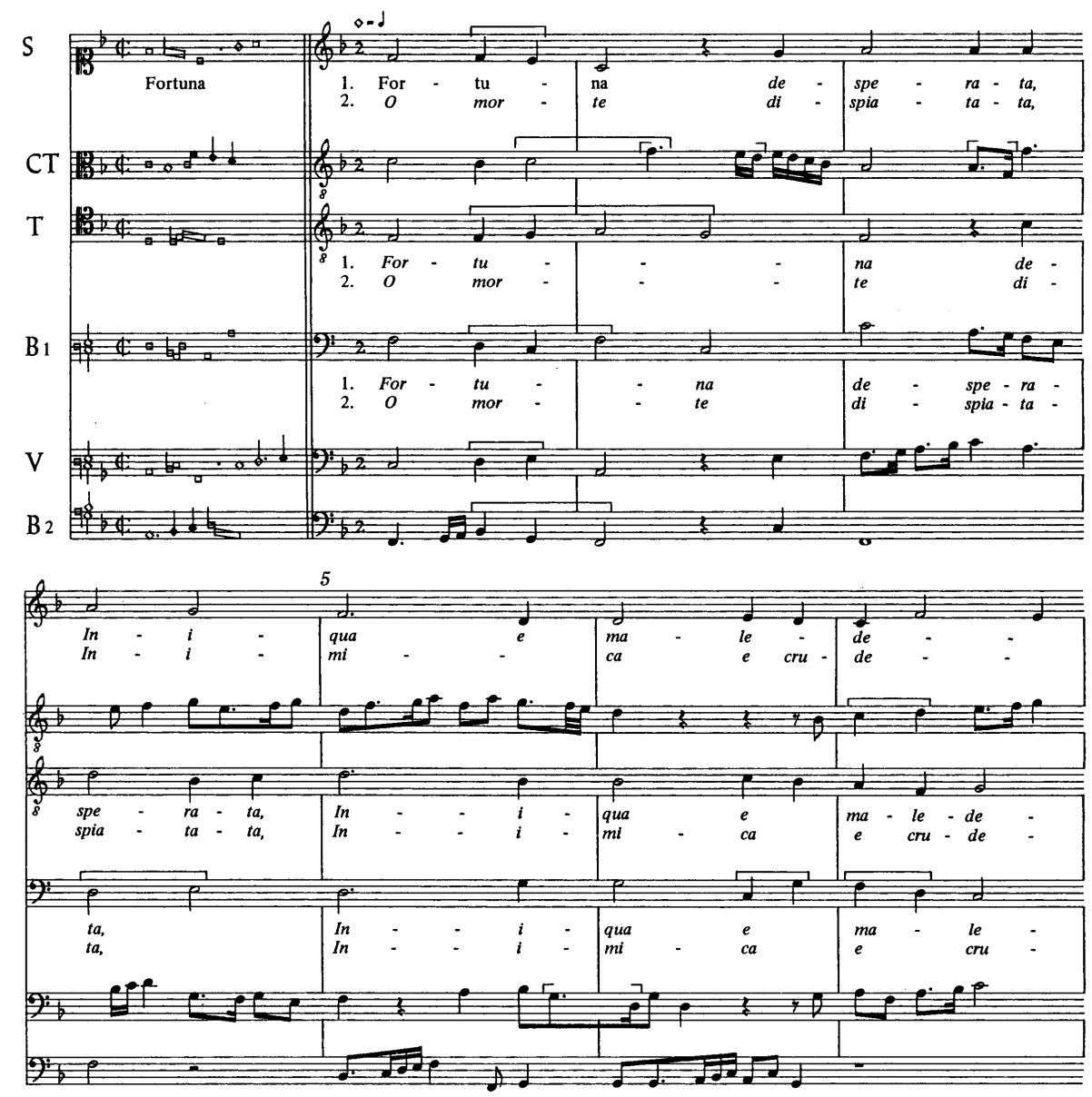

Notenbeispiel 1: Alexander Agricola, »Fortuna desperata«, Beginn ${ }^{4}$

Notengruppen. Der Ambitus der neuen Stimmen ist nicht außergewöhnlich groß. Bewegen sich die Stimmen des Kernsatzes (Superius, Tenor und Bassus 1) innerhalb einer None, so greifen die hinzugefügten Stimmen nur eine

4 Aus: Alexandri Agricola Opera omnia, hrsg. von Edward R. Lerner, Bd. 5: Cantiones, Musica Instrumentalis, Opera dubia, American Institute of Musicology 1970 (Corpus Mensurabilis Musicae, 22.5), Nr. 47, S. 68. Die untextierten Stimmen sind die »si placet«-Stimmen. 
Terz weiter aus, bleiben also im Rahmen einer Undezim (Oktave plus Quarte). Der Klangraum, der ursprünglich von $c$ bis $d$ " reichte, spannt sich durch die Erweiterung auf $F$ bis $d$ ”, bereichert also vor allem in der Tiefe und ist insgesamt dichter.

Erhalten hat sich diese in der Anlage ungewöhnliche sechsstimmige Komposition in einer einzigen Quelle, dem so genannten Augsburger Liederbuch, einem »Gsangbuch in 4 voces « (so am Außendeckel notiert) aus dem frühen 16. Jahrhundert. ${ }^{5}$ Vermutlich steht diese Handschrift in engem Bezug zur Hofkapelle Kaiser Maximilians sowie den Kaiser-Städten Innsbruck und Augsburg, angelegt zur Sicherung des Bestandes. ${ }^{6}$ Die Nähe zu Ulrich Brätel ist augenfällig: Er stammt sehr wahrscheinlich aus Augsburg, hat in Wien studiert und stand in Kontakt mit dem Humanistenkreis um Joachim Vadian. $^{7}$

Ein Blick in die chorbuchartige Aufzeichnung der Stimmen (siehe Abbildung 1 auf den folgenden Seiten) hebt die Unterscheidung von Alt und Neu deutlich hervor: Links wurden die ursprünglichen Stimmen notiert, in der rechten Hälfte die neu dazu komponierten. Text gibt es hier keinen, die Textmarke »Fortuna« (links, unterhalb des ersten Systems) bzw. »Fortuna desperata « (rechts, etwa in der Mitte der Seite) dient der Identifizierung des Satzes und mag bei den Benützern der Handschrift die dazugehörigen Worte innerlich abgerufen haben.

Der Text selbst geht zurück auf eine Sammlung von italienischsprachigen Gedichten aus den 1470er-Jahren, die aus Florenz stammt und in der Wahl der Muttersprache der traditionellen Dichtersprache Latein bewusst eine Ab sage erteilt. In den drei Strophen wird von dem >verzweifelten Glück « (fortuna desperata «) - also dem Unglück ohne Hoffnung - gesprochen, das durch

5 Das Augsburger Liederbuch. Faksimile-Ausgabe ... nach dem Exemplar Sign. CIM $43\left(2^{\circ}\right.$ Cod. 142a) der Staats- und Stadtbibliothek Augsburg, Stuttgart 1997 (Faksimile-Edition Augsburg, 3); Neuausgabe von Luise Jonas, Das Augsburger Liederbuch. Die Musikhandschrift $2^{\circ}$ Codex 142a der Staats- und Stadtbibliothek Augsburg. Edition und Kommentar, 2 Bde., München 1983 (Berliner musikwissenschaftliche Arbeiten, 21), Bd. 2, Nr. 51, S. 127-131.

6 Rainer Birkendorf, Der Codex Pernner. Quellenkundliche Studien zu einer Musikhandschrift des früben 16. Jahrhunderts (Regensburg, Bischöfliche Zentralbibliothek, Sammlung Proske, Ms. C 120), 3 Bde., Augsburg 1994 (Collectanea Musicologica, 6), Bd. 1, S. 97-99.

7 Martin Bente (Hans Albrecht), Art. »Brätel«, in: Die Musik in Geschichte und Gegenwart, 2. Aufl., hrsg. von Ludwig Finscher, Personenteil, Bd. 3, Kassel und Stuttgart 2000, Sp. $760-764$. 
den Tod der Auserwählten ausgelöst wurde (Beginn der zweiten Strophe: $\gg \mathrm{O}$ morte $\ll)$ und in einer einzigen Wehklage seinen Ausdruck findet. ${ }^{8}$
Fortuna desperata,
Iniqua e maledecta,
Che de tal dona electa
Verzweifeltes Schicksal,
widerwärtig und verflucht,
La fama hai denigrata.
das den guten Namen
O morte dispietata,
einer hervorragenden Frau verdunkelt hat.
Inimice e crudele,
O erbarmungsloser Tod,
Che d'alto più che stelle
L'hai cusi abassata.
feindlich und grausam,
der du sie, die höher als die Sterne stand,
so erniedrigt hast.
Meschino et despietata
Ben piangere posso may;
Et desiro finire
Einsam und verzweifelt
kann ich nicht anders als weinen,
li mei guay.
und ich sehne mich nach dem Ende
meines Kummers. ${ }^{9}$

Gehen wir nochmals zurück handschriftlichen Quelle (siehe Abbildung) und richten wir das Augenmerk auf typische Charakteristika der Stimmführung, die man gemeinhin dem >Instrumentalen< zuweist. Dazu gehören in erster Linie kleinrhythmische Passagen mit einer Folge von Notenwerten wie Minimae und Semiminimae (inklusive kolorierter Noten). Ein Vergleich mit rechter und linker Manuskriptseite - also alten und neuen Stimmen - zeigt, dass rechts zwar häufig Passagen dieser Art auftreten, jedoch auch die linke Seite nicht frei davon ist. ${ }^{10}$ Auch große Instrumentalsprünge im Umfang von mindestens einer Septime kann man bei Alt und Neu feststellen, rechts allerdings doppelt so viele wie links. ${ }^{11}$ Was also im Kernsatz schon angelegt ist, wird in den Zusatzstimmen noch bewusst verstärkt und ins Extrem getrieben. Also instrumentale Ausführung?

\section{Hören: Vergleiche}

Die moderne Aufnahmetechnik und ein florierender Musikmarkt machen es möglich, nicht nur eine qualitätvolle Einspielung von »Fortuna desperata« zur Verfügung zu haben, sondern gleich drei verschiedene. Da jede dieser Aufnahmen eigen ist in der Besetzung und Interpretation, lohnt ein genauer

8 Honey Meconi, »Poliziano, >Primavera $<$, and Perugia 431: New Light on $>$ Fortuna desperata< «, in: Antoine Busnoys (wie Anm. 2), S. 465-503.

9 Übersetzung nach www.cpdl.org (free choral sheet music).

10 Auf der rechten Blatthälfte zählt man 37 solche Passagen (davon vier sogar mit Fusae), auf der linken zwei.

11 Genau gezählt ist das Verhältnis 4:2. 

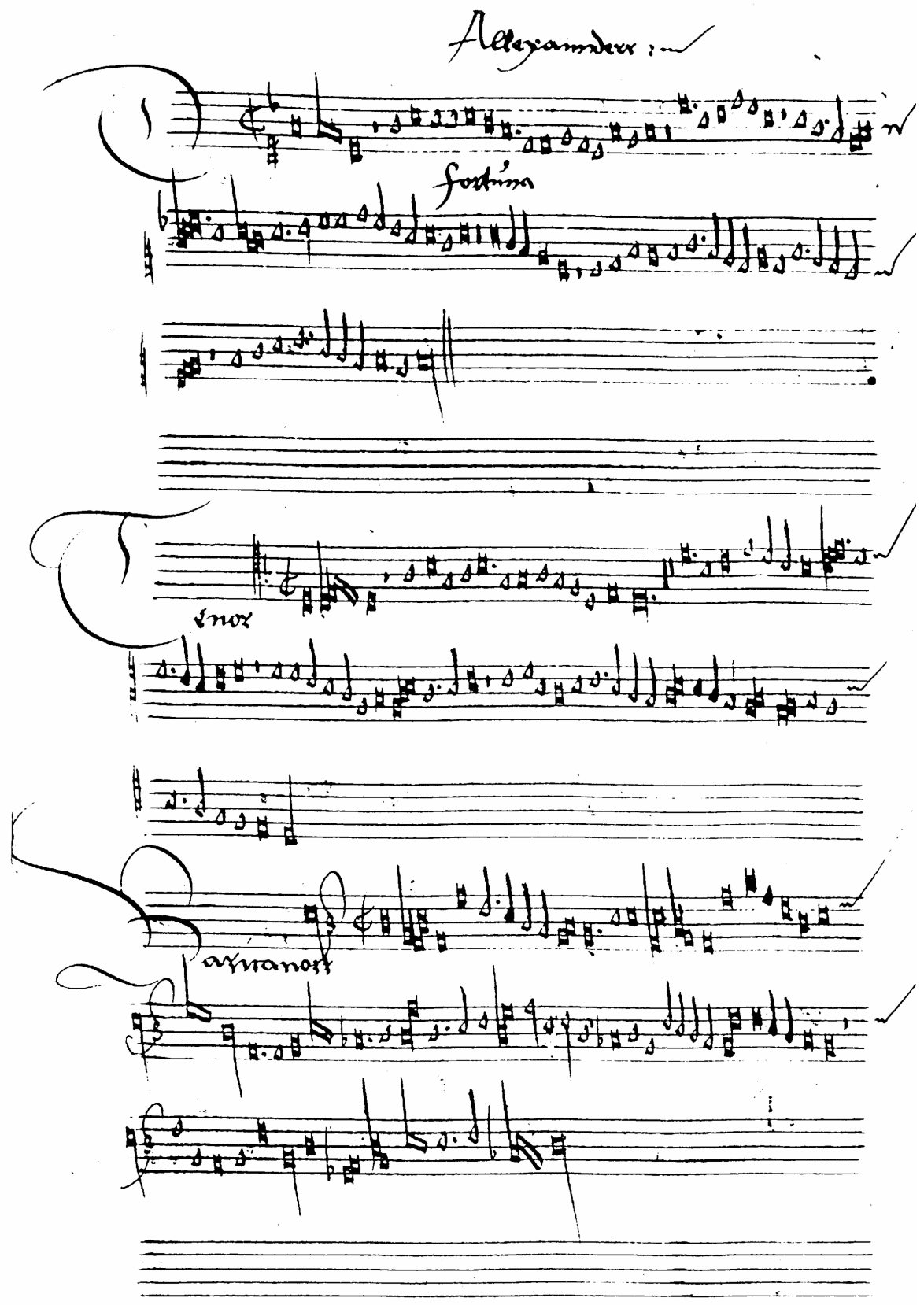

Abbildung 1: Alexander Agricola, »Fortuna desperata« im Augsburger Liederbuch 


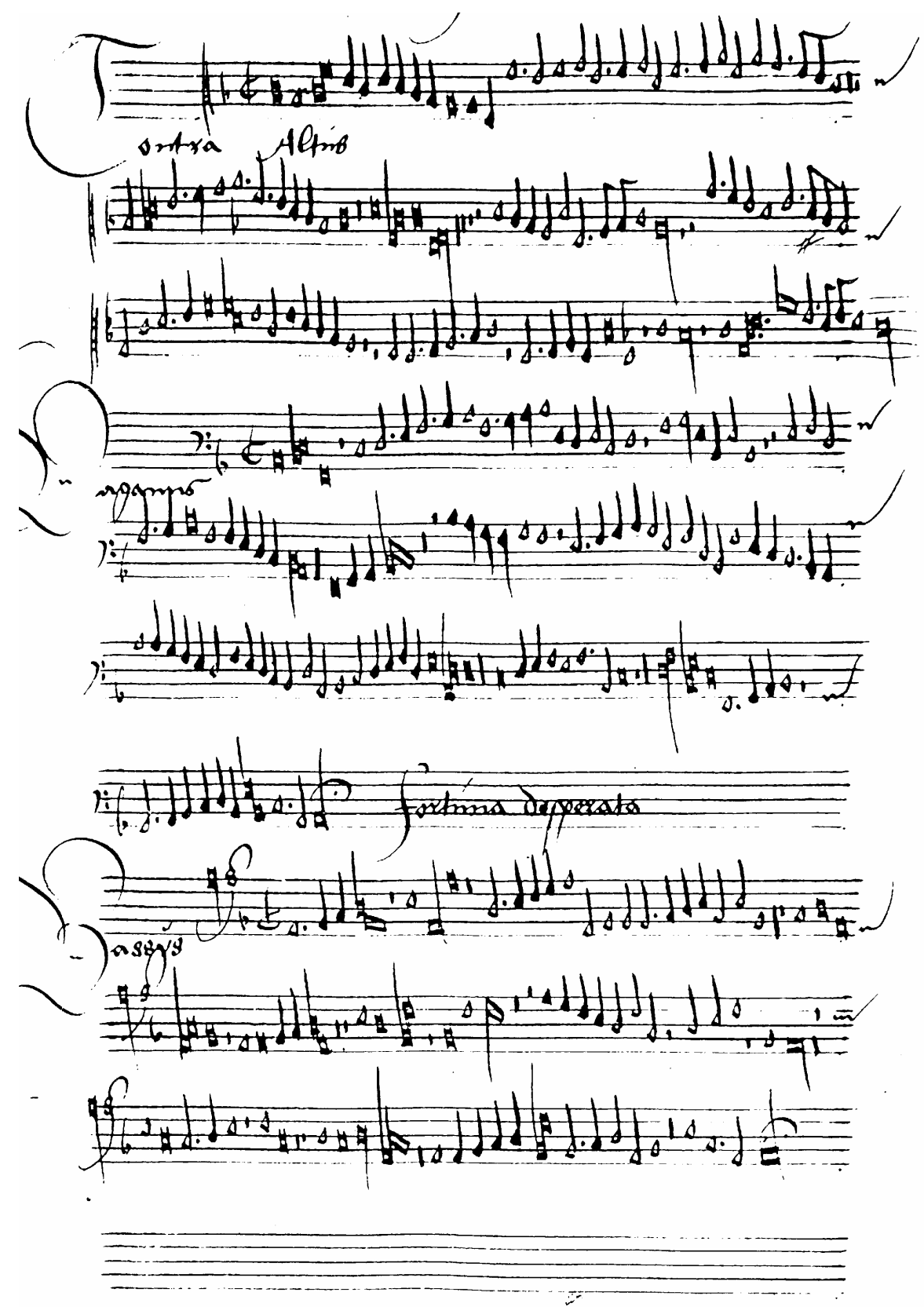

(D-As, $2^{\circ} 142 a$, fol. $46^{v}-47^{r}$ ) 
Vergleich. ${ }^{12}$ Bei den CD-Aufnahmen, allesamt Einspielungen der 1990erJahre, handelt es sich um:

1. Alexander Agricola: Fortuna desperata. Secular Music of the 15th Century. Ensemble Unicorn, Michael Posch. Naxos (Early Music/Alte Musik) 1995

2. A. Agricola. Chansons. Ferrara Ensemble, Crawford Young. Deutsche harmonia mundi 1990

3. Alexander Agricola (1446-1506). A Secret Labyrinth, Huelgas Ensemble, Paul van Nevel. Sony Classic (music for you) 1998

Die Aufnahmen sind vor allem hinsichtlich der Besetzung grundverschieden. Bei Nr. 1 (Ensemble Unicorn) finden wir eine gemischte Besetzung, wobei der $>$ Ursatz $<$ vokal, die hinzugefügten Stimmen instrumental ausgeführt werden; bei Nr. 2 (Ferrara Ensemble) werden alle Stimmen mit Saiteninstrumenten besetzt, gezupft und gestrichen; und bei Nr. 3 (Huelgas Ensemble) hören wir einen rein vokalen Satz in drei Strophen.

Der folgende Interpretationsvergleich orientiert sich an jenen Fragen, die Nicole Schwindt in ihrem Einführungstext zur Tagung formuliert hat:

- Wie funktioniert die Mischung unterschiedlicher klanglicher Idiome? Entstehen gerade daraus neue ästhetische Qualitäten?

- Welche Konsequenzen hat die stilistische Überblendung, wenn man die Musik in unterschiedlichen Besetzungen realisiert?

- Kann man daraus womöglich objektive und nachvollziehbare Optionen und Empfehlungen für konkrete Ensemblezusammenstellungen ableiten?

Beim Hören geht es zunächst nicht um diese differenzierten Fragen, sondern um einen anderen, grundlegender Parameter, der sich als erster starker Eindruck in den Vordergrund drängt: das unterschiedliche Tempo. Die mittlere, rein instrumentale Interpretation von Crawford Young und seinem Ensemble ist etwa um die Hälfte schneller als die beiden vokalen Aufnahmen ${ }^{13}$ und vermittelt schon allein dadurch eine ganz andere Stimmung. Durch den Einsatz von gezupften Instrumenten in den oberen vier Stimmen wirkt der

12 Im Vortrag wurden alle drei Aufnahmen nacheinander gespielt und mit dem Publikum gemeinsam diskutiert. Die folgenden Erkenntnisse sind von diesen Wortmeldungen geleitet. Ich bedanke mich für alle Beiträge.

13 In Metronomzahlen gemessen und auf die Semibrevis bezogen hat Nr. 1 MM 68, Nr. 2 MM 96 und Nr. 3 MM 69. Die zeitliche Länge einer Strophe beträgt entsprechend 1:53, 0:57 bzw. 1:54 Minuten. 
Satz hier dünner, das Tempo muss so schnell genommen werden, um die Linien nicht abreißen zu lassen. Zudem treten durch die spezifische Besetzung die schnellen Noten der ergänzten Stimmen sehr viel stärker hervor und füllen gleichsam die klanglichen Lücken, die beim Zupfen der Stimmen des >Ursatzes< entstehen. Ein leicht vituos-instrumentales Moment, das man besser als manuelle Spielfreude bezeichnen möchte, ist dieser Interpretation zu Eigen.

Beinahe ein anderes Stück meint man zu hören, wenn unmittelbar anschließend die dritte Aufnahme mit der Interpretation des Huelgas Ensembles unter Paul van Nevel erklingt. Der leichte >drive< der vorangegangenen Einspielung weicht einem getragenen, sensitiven Vokalsatz, der aus einer völlig anderen Welt kommt. Das Ohr sucht nach den schnellen rhythmischen Spielfiguren und findet sie als sanftes Gleiten der Vokalstimmen wieder. Man ist beeindruckt von dem hohen stimmtechnischen Niveau und erlebt die klanglichen Spannungen, die durch die Feinintonation der Stimmen ermöglicht wird, als neue Qualität der Komposition.

Geht man nun wiederum auf die erste Interpretation des Ensembles Unicorn unter der Leitung von Michael Posch zurück, so stellt sich ein interessanter Effekt ein. Obwohl beide Einspielungen gleich lang sind, wirkt jene mit der vokal-instrumental gemischten Besetzung um einiges schneller. Dieser Eindruck dürfte mit der viel offeneren Stimmgebung der Sänger zusammenhängen, aber auch mit dem direkten Zugriff auf den Notentext, also eigentlich einer ästhetischen Haltung. Die Tatsache, dass die drei »si placet«Stimmen von Agricola mit Streichinstrumenten besetzt sind, scheint dabei nur indirekt eine Rolle zu spielen. Der Klang der Fidel und der beiden Vihuelas d'arco ist gegenüber den dominierenden Gesangsstimmen stark zurückgenommen, sie untermalen eher den $>$ Ursatz $<$ als dass sie ihm etwas Gleichwertiges entgegenstellen. Außerdem wirkt der Vokalsatz gegenüber der vokalen Aufnahme lockerer.

Damit kommen wir schon zur ersten Frage, die sich aufgrund dieser drei unterschiedlichen Interpretationen aufdrängt: nämlich, wie weit soll man analytisch besetzen, bzw. wie weit soll man mit der Besetzung ein analytisches Hören unterstützen? In der eben genannten Einspielung von Unicorn ist dies offensichtlich ein Anliegen, das durchaus legitim ist. Allerdings wurde dabei, wie erwähnt, keine klangliche Ausgewogenheit erzielt und damit die Bedeutung der kompositorischen Leistung Agricolas - die man angesichts der Vielzahl der verschiedenen Vertonungen von »Fortuna desperata« zweifellos im Hinzusetzen der »si placet«-Stimmen sehen muss - heruntergespielt. Bei den anderen beiden Einspielungen wird keine der sechs Stim- 
men durch eine spezifische Besetzung bevorzugt, meiner Ansicht nach ein genauso interessanter Zugang, der den vollstimmigen Satz ins Zentrum stellt und somit >Ursatz< und Ergänzungen als neue Einheit erscheinen lässt.

Grundsätzlich bin ich der Meinung, dass Einzelstimmen, die als Grundlage einer neuen Komposition dienten (man denke etwa an die unterschiedlichen Vertonungen Agricolas von »De tous biens plaine«), sehr bewusst auf den Gesamtklang hin besetzt werden sollten. Geht man von einem Publikum aus, das die Lied- oder Choralmelodie nicht kennt, dann erscheint es angemessen, die betreffende Stimme so zu besetzen, dass sie klanglich mitverfolgbar, vielleicht sogar dominant erklingt. Diffiziler ist es freilich, wenn man mit der Bekanntheit einer Melodie spielen und gerade durch die Besetzung andere Klanglichkeiten der Komposition hervortreten lassen kann, als man es durch die etwas platte Standardinterpretation mit verstärkter $>U r-$ stimme gewohnt ist. ${ }^{14}$ Das Neue im Alten - um wieder auf unser Generalthema zurückzukommen - ist immer ein besonderer Reiz. Und gerade das hat ja auch die Komponisten des 15. und 16. Jahrhunderts dazu veranlasst, aus einem bestehenden, bewährten Satz Stimmen herauszulösen und daraus Neues zu gestalten.

Schließlich sei noch das Problem der Textbedeutung und Textausdeutung anhand der drei gewählten Interpretationsbeispiele angesprochen. Nur in der letzten, rein vokalen Aufnahme des Huelgas Ensembles wird die Klage über die tote Geliebte durch die Gehaltenheit der Stimmen, gekoppelt mit dem langsamen Tempo, im ästhetischen Gesamteindruck unmittelbar vermittelt. Auch ohne den Text zu kennen (oder zu verstehen), erfasst man intuitiv die emotionale Aussage und erinnert sich an Totenklagen von Komponisten, die auch Ulrich Brätel in seinem Liedtext nennt: Josquins »Nymphes des bois« und Ockeghems »Mort tu as navré«. Agricolas »Fortuna desperata « in der Vokalinterpretation hat viel stärker motettischen Charakter als eine Komposition, die als schlichtes $>$ Lied $<$ verstanden sein will. Dieser Eindruck wird noch unterstützt durch einzelne Passagen mit forcierter Textausdeutung, die mit Hilfe von Musica ficta bzw. dem Verzicht darauf zustande kommen. Insbesondere die Textzeile mit den Worten »maledecta« (1. Stro-

14 Das gilt auch für ganz andere Repertoires, wie etwa der klassischen Klaviermusik. Beispielhaft findet man diesen Zugriff in den Interpretationen von Andras Schiff, der bei wiederholten Passagen gerne Melodielinien der linken Hand hervorhebt und dadurch in einer meist bestens bekannten Komposition Neues hervorzaubern kann. 
phe) bzw. »crudele « (2. Strophe) erfährt in der Interpretation von van Nevel und seinem Ensemble in Takt 10 eine für das Ohr wirklich "grausame « Intervallfolge $\left.G-g-d^{\prime}-b^{\prime} / g-b-d^{\prime}\right) / F-a-c^{\prime}-c^{\prime}$ (siehe Notenbeispiel 2). Die beiden anderen Aufnahmen spielen hier, wie auch die Ausgabe im Corpus Mensurabilis Musicae andeutet, ein b-Vorzeichen im Bassus I. Der Charakter einer Totenklage geht bei der Instrumentalaufnahme völlig verloren, in der gemischten Aufnahme ist er vorrangig durch das Tempo, weniger durch den klanglichen Eindruck vermittelt.

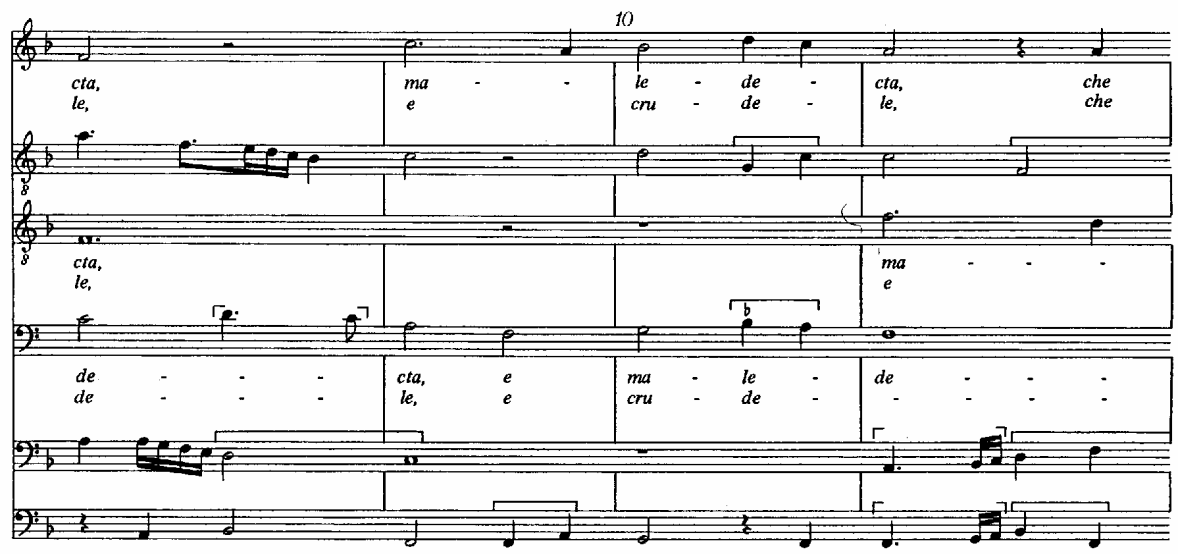

Notenbeispiel 2: Alexander Agricola, »Fortuna desperata«, Takt 8-11 $11^{15}$

Die Textvorlage einer Komposition bietet nicht nur einen Anstoß zur inhaltlichen Interpretation, sondern hat auch gliedernde Funktion, zum einen bezüglich der Abschnittsbildung, zum anderen aber auch die Großform betreffend. ${ }^{16}$ Ein Lied mit drei Strophen, so meint man, sollte dreimal hintereinander, jeweils mit anderem Text, vorgetragen werden. Aber keine der drei Einspielungen hält sich an dieses Prinzip. Bei der gemischten Besetzung kannte man vielleicht nur den Text der ersten Strophe und ein einmaliger Durchlauf genügte. Bei der instrumentalen Interpretation scheint es wieder-

15 Wie Anm. 4, S. 69.

16 Siehe dazu die anregenden theoretischen Überlegungen von Leeman L. Perkins, »Towards a Theory of Text-Music Relations in the Music of the Renaissance «, in: Binchois Studies, hrsg. von Andrew Kirkman und Dennis Slavin, Oxford 2000, S. 313-329. 
um wenig sinnvoll, textlos dreimal das Gleiche zu spielen. Aus anderen musikalischen Gründen hat man sich hier jedoch entschlossen, zumindest eine Wiederholung auszuführen und fügte beim fiktiven Strophenwechsel eine nette Übergangsfloskel ein. Die dritte, rein vokale Aufnahme schließlich bringt zwar tatsächlich alle drei Strophen, spielt dabei aber mit der >Urform< als gestalterischem und klanglich reduziertem Element. Sie erklingt als Mittelteil einer symmetrischen Formgestalt mit dem Text der zweiten Strophe.

\section{Schlussbemerkungen}

Der Vergleich der drei Einspielungen sollte keinesfalls zeigen, welche die beste oder gar >die richtige< Interpretation ist. Vielmehr war es wichtig, im bewussten Hören nachzuvollziehen, wie sehr sich der Werkcharakter durch Besetzung - aber auch durch individuelle Interpretation - verändern kann, und welche Möglichkeiten sich hier eröffnen. Praktikern Empfehlungen für konkrete Ensemblezusammenstellungen zu geben, wäre nicht Sinn der Sache, sind es ja gerade die Vielfalt der Besetzungsvarianten und die daraus erwachsenen musikalischen Konsequenzen, die das weltliche Repertoire der Zeit so reich erscheinen lassen.

Abschließend soll neben den vokal-instrumentalen Ensemble-Interpretationen noch eine weitere Besetzungsvariante erwähnt werden, nämlich die der Intabulierungen. Sie schwinden allzu leicht aus dem Blickfeld, sind sie doch in eigener Notenschrift festgehalten, für das Instrument eingerichtet und oft nur einer schmalen Gruppe von Spezialisten bekannt. Meines Erachtens aber sollten sie in einer wissenschaftlichen Ausgabe als weitere Erscheinungsform einer Komposition ihren Platz gleichberechtigt neben der Ensemblefassung einnehmen und auch in Reihen wie den Corpus Mensurabilis Musicae aufgenommen werden - auch wenn ihre Notationsform dem Reihentitel widerspricht. Ebenso wäre es längst an der Zeit, in den Sammeleditionen die kategoriale Unterscheidung zwischen Instrumental- und Vokalwerken fallen zu lassen, denn gerade damit untergräbt man den Schwebezustand der Kompositionen zwischen Vokal und Instrumental, und damit auch das interessante Wechselspiel zwischen Alt und Neu. 